\title{
Editorial: Biodegradable Matrices and Composites
}

\author{
Alessandro Pegoretti ${ }^{1 *}$, Yu Dong ${ }^{2}$ and Miroslav Slouf ${ }^{3}$ \\ ${ }^{1}$ Department of Industrial Engineering, University of Trento, Trento, Italy, ${ }^{2}$ School of Civil and Mechanical Engineering, Curtin \\ University, Perth, WA, Australia, ${ }^{3}$ Institute of Macromolecular Chemistry, Czech Academy of Sciences, Prague, Czechia
}

Keywords: biodegradable polymers, biodegradable composites, biodegradability, material characterization, environmental sustainability, recyclability

\section{Editorial on the Research Topic}

\section{Biodegradable Matrices and Composites}

Biodegradable and ecofriendly materials attract an increasingly high attention from polymer and composite researchers and manufactures as they can contribute to a more sustainable global development policy, to significantly reduce non-recyclable plastic wastes and limit the impact on the environment. A thorough understanding of both advantages and limitations of biodegradable matrices and composite materials is the key to their reasonable, precisely targeted and effective applications in order to benefit wide communities. The attention in the field of real applications is focused mostly on packaging materials, but automotive, construction, and agriculture are also important fields. As for medical applications, biodegradable matrices and composites are used mostly due to their tunable biodegradability, which is often combined with controlled and localized drug release. The term of biodegradable composites comprises a wide range of at least two-phase hybrid materials in which either fillers or matrix or both must be chosen from biodegradable sources. Hence, those matrices based on biodegradable polymers are also well-recognized as biodegradable matrices. On the other hand, fillers being the minority phase act as a reinforcement in most biodegradable composites. However, with respect to polymer blends, the minority phase often plays a different role as an impact modifier, a plasticizer or a compatibilizer, as opposed to its drug carrier status in medical applications. Particular cautions should always be taken in the case of composites containing synthetic polymers as matrices, whose biodegradability, biocompatibility, and/or environmental impact may be rather questionable. On the other hand, composites containing bio-based biodegradable polymers as matrices can be fine-tuned to exhibit good tensile, impact and creep properties in spite of lower resistance to moisture, leading to poor mechanical performance at high humidity or aqueous conditions.

The Research Topic on Biodegradable Matrices and Composites is focused on recent advances in preparation and characterization of biodegradable polymeric matrices and their composites with the main objective to understand their processing-structure-property relationship at nano-, micro-, and macroscale. Both conventional biodegradable polymers and related biodegradable composites and more recent biodegradable nanocomposites reinforced with nanoparticles and nanofillers are covered to address various aspects, including but not limited to their mechanical, thermal and barrier properties, biodegradability, environmental sustainability, renewability and recyclability, limitations and applications in building constructions, automobiles, medical devices, and material packaging.

The edited book for this Research Topic comprises a special issue collection of 24 contributions represented by 1 mini review, 3 full review, 1 perspective, and 19 original research articles, which are classified into Part A: Biodegradable Polymers, Blends, and Matrices and Part B: Biodegradable Composite Materials. In Part A, Mistretta et al. investigated mechanical, structural and optical properties of polylactic acid (PLA), polybutylene adipate terephthalate (PBAT), and 
PBAT/PLA blends, as well as their surface properties and water vapor permeability before and after the exposure to UV irradiation in order to evaluate their durability and suitability for the conservation of culture heritage. Apicella et al. developed a sustainable food-packaging solution with antimicrobial effectiveness and high functional performance by spreading PLA/ Ethyl-N $\alpha$-dodecanoyl-L-arginate (LAE) coating solution on a recyclable polyethylene-terephthalate (PET) substrate. E. coli CECT 434 strain as a pathogenic agent was employed to test antimicrobial activity of such multilayer films in liquid culture media. Ten percentage of LAE was found to be the minimum concentration to warrant total inhibition without significantly altering the functionalities of the developed systems. Iozzino et al. prepared biphasic samples (half amorphous and the other half crystalline) of PLA using micro-injection molding. Hydrolysis tests were carried out to monitor their degradability, which indicated that crystalline regions exhibited slightly better resistance to the hydrolysis as opposed to amorphous phase. Fortelny et al. reported a comprehensive review on morphology, compatibility, and mechanical properties of PLA/ poly ( $\varepsilon$-caprolactone) (PCL) blends in order to control blend toughness and crystallinity so that the blending of PLA and PCL can result in desirable materials with well-tailored mechanical properties. Among other things, the review showed how to prepare super-tough PLA/PCL blends without compatibilization. Nevoralová et al. evaluated the effect of blending PCL with thermoplastic starch (TPS) on the final biodegradation rate of such blends in both compost and soil environments. Very fast biodegradation with the initial similar rate to that of pure TPS in both environments was clearly identified for the blends containing 70\% TPS with a co-continuous morphology. Whereas, 30\% TPS blends demonstrated the particle morphology of starch phase in PCL matrix, indicating a dominant effect of the matrix on the biodegradation course. Delamarche et al. investigated the relationships between physical properties and tailored biodegradability for poly(butylene succinate) (PBS)/PLA blends using a deep eutectic solvent (DES). It was shown that molar masses, crystallinity, yield, morphologies, and surface properties were crucial to control the biodegradability of blend materials. Aliotta et al. studied a multiphase ternary system comprising two different elastomers of PBAT and polyolefin elastomer grafted with glycidyl methacrylate (POE-g-GMA) being added into PLA matrix, as compared with PLA and PLA/PBAT binary blends with maximum $10 \mathrm{wt} \%$ PBAT. It was found that the best compromise between impact, tensile properties and biodegradability content could be achieved by using a reactive plasticizer EJ-400. Rigotti et al. evaluated the effect of blending poly(3-hydroxybutyrate) $[\mathrm{P}(3 \mathrm{HB})]$ to linear low density polyethylene (LLDPE) at the $\mathrm{P}(3 \mathrm{HB})$ concentrations in the range of $10-50 \mathrm{wt} \%$ on hydrolytic degradation, water absorption, thermal and mechanical properties as a function of hydrolysis time. It was demonstrated that the use of biodegradable plastic $\mathrm{P}(3 \mathrm{HB})$ at a relative amount of $20 \mathrm{wt} \%$ to substitute for LLDPE could successfully develop a desirable material in order to meet processability requirements with better tailored mechanical properties even after prolonged hydrolytic degradation treatment. Neisiany, Enayati, Kazemi-Beydokhti et al. provided a mini review on current state-of-art development of multilayered biodegradable electrospun membranes along with new insights into the future of tailored membranes toward practical applications. Mohammadzadehmoghadam and Dong investigated the impact of gelatin content on material properties and structures of silk fibroin (SF) nanofibers crosslinked with glutaraldehyde vapor (GTA) to understand the potential application of such biodegradable material matrices in tissue scaffolding. The detailed characterization was performed by scanning electron microscopy (SEM), Fourier transform infrared spectroscopy (FTIR), mechanical testing, degree of crosslinking test, water uptake test, water contact angle measurements, X-ray diffraction (XRD) analysis, as well as biocompatibility assessment using fibroblast cells. Adverse effect of GTA crosslinking was detected to reduce the capacity of supporting the cell activity despite improved mechanical properties of nanofiber mats. GTA optimization became essential to further modulate physicochemical properties of SF/gelatin nanofiber mats in order to achieve stable materials with favorable bioactive properties and more active cellular response in tissue engineering. Zaidi et al. reported the incorporation of epoxidized natural rubber (ENR) as a biodegradable toughening agent, which could enhance toughness properties of polyhydroxybutyrate-covalerate $(\mathrm{PHBV})$ biodegradable composites and also improve their biodegradability in a more substantial manner, when compared with the addition of commonly used toughening agent PBAT.

In Part B, Aini et al. presented the detailed review with respect to the performance of lignin-filled rubber composites using different approaches including mixing methods, surface modification hybrid fillers and so on in order to gain the insights to the advances in the development of green rubber products. Girijappa et al. thoroughly reviewed different sources of natural fibers, their properties as well as the effect of modification and treatments on natural fibers, etc. with major applications targeting the reinforcements for polymer composites. Salim et al. investigated the effect of fiber surface treatments (i.e., alkali treatment, alkali treatment at elevated temperature, and hear treatment) on the durability of kenaf fiber reinforced acrylic based polyester composites under accelerated weathering exposure for potential applications in automotive industry. Moreover, the excellent fiber-matrix adhesion associated with these treatments may also decrease the water absorption rate by such composite materials. Aouat et al. assessed the influence of combined humid atmosphere and temperature on the morphology, chemical structures, and physical properties of neat PLA, PLA/PLA-grafted-maleic anhydride (PLA-g-MA)/microcrystalline cellulose (MCC) and PLA/PLA-g-MA/cellulose nanowhisker (CNW) biodegradable composite fibers. It has been found that the durability of PLA fibers to hygrothermal degradation can be established in the following order: PLA > PLA/PLA- $g$-MA/MCC > PLA/PLA$g$-MA/CNW where the MCC and CNW concentrations are 1 wt $\%$ and PLA- $g$-MA content is 7 wt $\%$. Slouf et al. successfully prepared biodegradable microfibrillar PCL/PLA/organophilic montmorillonite composites. It was reported that the macroand micromechanical properties of all intermediate products and final microfibrillar composites were related not only to the composition and morphology, but also to the crystallinity of 
both components. In particular, the modulus of such composites reached almost twice higher value in comparison with the original PCL matrix. Li et al. provided a physical and chemical analysis of chicken feather (ChF) biocarbon generated at different pyrolysis temperatures, which were further combined with PLA matrix to generate $100 \%$ sustainable composites. The authors analyzed their thermal properties, mechanical properties, and composition. The valorized $\mathrm{ChF}$ as sustainable natural fillers can be a safe and suitable alternative used for composite applications in order to reduce their waste disposal. Ujcic, Krejcikova et al. demonstrated a two-step preparation of TPS using both solution casting and melt mixing to prepare TPS/ $/ \mathrm{TiO}_{2}$ composites with homogeneous filler dispersion even at high filler concentrations up to $20 \mathrm{wt} \%$. The same approach was also employed to uniformly disperse antibiotic (ATB) vancomycin at $10 \mathrm{wt} \%$ in TPS systems. The interactions among TPS, $\mathrm{TiO}_{2}$ particles, and ATB molecules gave rise to the shift of glass transition temperatures to higher values along with stiffening effect on corresponding composites. It has been proven through standard antimicrobial susceptibility tests that neither thermal processing nor the addition of $\mathrm{TiO}_{2}$ influence the ATB, which remains active in both TBS/ATB and TPS/TiO 2 /ATB composites. Fully biodegradable TPS composites are believed to be very promising materials for biomedical applications with respect to local release of antibiotics. Ferrari et al. developed new green composite material with the incorporation of natural additives such as shredded walnut shells and organic fraction of municipal solid waste (MW) into poly(vinyl) alcohol (PVA) for the improvement of mechanical properties of composites. The addition of walnut shells indicated the increases in glass transition temperature and flexural modulus reached about 50\% along with the reduction in flexural strength by $\sim 30 \%$. Furthermore, when added with a low amount of water, the inclusion of MW resulted in increasing strain at break by $100 \%$. Bugatti et al. evaluated the recovery and upgrade of tomato processing residues to be used for the manufacture of innovative green composites based on tomato peels (TPs) and natural halloysite nanotubes (HNTs) loaded with carvacrol as a natural antibacterial agent. Thermal properties of such biodegradable composites were improved with increasing the filler loading as opposed to worse mechanical properties, which especially took place at higher loading levels associated with the poor interaction between HNTs and matrix. No dramatic increase was detected in the degree of hydrophilicity of biodegradable composites. This phenomenon might be attributed to the role of hydrophobic carvacrol acting as hydrophobic coating for HNTs. Finally prepared biodegradable composites exhibited a long-term release of antimicrobial agents, greatly benefiting their wide applications in food packaging. Abdullah and Dong mentioned the comprehensive material development of PVA/starch (ST)/HNT biodegradable composite films for sustainable packaging. Such films possessed high water resistance, good biodegradability, acceptable transparency, as well as overall migration rates to target sustainable food packaging particularly for lipophilic and acidic foodstuffs. Ujcic, Nevoralova et al. compared wheat and tapioca TPS and their composites with $\mathrm{TiO}_{2}$-based nanoparticles (i.e., isometric $\mathrm{TiO}_{2}$ nanoparticles and high-aspect-ratio titanate nanotubes) where
TPS composites were prepared by a combined technique using solution casting and melt mixing. Such a combined technique was proven to be effective to the manufacture of TPS composites with homogeneous filler dispersion regardless of starch sources and types. The type of TPS matrix (i.e., wheat or tapioca) was shown to have significant impact on the properties of resulting composites in which isometric $\mathrm{TiO}_{2}$ nanoparticles yielded very good filler dispersion while TiNT nanotubes tended to form microsized aggregates within both TPS matrices. Liu et al. successfully developed CNW/graphene nanoplatelet (GN) composite films with respect to the GN role as rigid fillers, and further evaluated filler-matrix interactions to concurrently improve their thermal, electrical, and mechanical properties, which were difficult to achieve in conventional nanocomposite materials. Such CNW/GN composite films are anticipated to be potentially effective multifunctional materials in the field of electronic packaging. Finally, Neisiany, Enayati, Sajkiewicz et al. briefly reviewed recently reported work on nanocomposite hydrogels based on chiral functionalized nanomaterials. This work can pave the way for the development of biodegradable hydrogels toward real practical applications.

In summary, this edited book offers a diverse range of biodegradable polymers, their blends and biodegradable composite materials, focusing on their fabrication, characterization and widespread applications. It can be deemed as a good overview for materials engineers and scientists, experienced researchers and postgraduate students, as well as industrial staff working in research \& development sectors to rapidly move forward to innovative and ecofriendly materials for significantly reducing conventional petroleum-based plastic wastes at disposal.

\section{AUTHOR CONTRIBUTIONS}

YD prepared the initial editorial draft while AP and MS provided further comments and feedback. YD, AP, and MS jointly finalized the editorial work. All authors contributed to the article and approved the submitted version.

\section{ACKNOWLEDGMENTS}

We would like to thank all the authors, reviewers and handling editors to make every success in this Research Topic. We are also indebted to the editorial team of Frontiers in Materials including Mina Roussenova, Paola Morelli, Alexander Stephens, and Michelle Cook to assist in every step from Research Topic proposal, article review and production to final publications.

Conflict of Interest: The authors declare that the research was conducted in the absence of any commercial or financial relationships that could be construed as a potential conflict of interest.

Copyright (C) 2020 Pegoretti, Dong and Slouf. This is an open-access article distributed under the terms of the Creative Commons Attribution License (CC BY). The use, distribution or reproduction in other forums is permitted, provided the original author(s) and the copyright owner(s) are credited and that the original publication in this journal is cited, in accordance with accepted academic practice. No use, distribution or reproduction is permitted which does not comply with these terms. 